\section{Need of Guidelines and Antimi- crobial Stewardship in Pakistan for Perioperative Surgical Practices to Prevent Surgical Site Infections}

Sir,

Globally, every year millions of surgical procedures are carried out. ${ }^{1}$ Surgical site infections (SSIs) occur at the site of surgery within 30 days or in one year, if any implant is placed. ${ }^{2}$ Nearly $20 \%$ of total surgeries are complicated by SSIs. ${ }^{1}$ According to the WHO report, about $66 \%$ of the developing countries have no published data on SSI. The associated risk factors can be controlled through proper care at the hospital by some guidelines. Four types of guidelines/criteria (CDC, MUST, ASA and KUNIN) may help prevent the SSI incidence. Malnutrition is an important contributing risk factor, and can be predicted through MUST (malnutrition universal screening tool). CDC (center for disease control and prevention) guidelines for classes of wounds, and ASA (American society of anesthesiologists) for physical status indication, would be helpful for the surgeons. ${ }^{3}$ KUNIN (rational antibiotics grades) is for the assessment of rational antibiotic prescription and might be helpful in the promotion of rational prescription. $^{1,3}$

The antimicrobial stewardship (AMS) programmes are mainly developed by physicians along with clinical pharmacists. ${ }^{4,5}$. The goal of AMS is to reduce antimicrobial resistance; and ultimately, the patient expenditures and length of stay at the hospital will decrease. ${ }^{1}$ The pharmacist interventions for the prescribed antibiotics and drug utilisation review may lead to improved treatment outcomes of the individual patient. ${ }^{5}$ Clinical pharmacists and surgeon's contributions to the therapy can reduce the incidence rate of SSIs.

Although Pakistan is facing a huge burden of infections after surgeries, it is still noticeable how this issue is being ignored. ${ }^{1,5}$ There is severe lack of research studies on this topic. Pakistan is still lacking in terms of effective AMS. The implementation of AMS programmes across the country is a need to prevent the incidence of SSIs in terms of the irrational use of antibiotics from hospitals to patients' homes. Pakistan had launched National Action Plan for Antimicrobial Resistance, butstill no fruitful results have been seen; and recently 'antimicrobial resistance' week was celebrated during October 2019. Proper perioperative guidelines along with effective AMS programmes at hospitals across the country and strict regulations are need of the hour in Pakistan to prevent the high rates of SSIs.

\section{CONFLICT OF INTEREST:}

Authors declared no conflict of interest.

\section{AUTHORS' CONTRIBUTION:}

FUK: Conceptualised the main theme of this letter and have played major role in the process of writing.

ZK: Helped in the literature review and editing.

AUR: Played key role in the writeup and editing.

YF: Supervised and approved the final draft.

\section{REFERENCES}

1. Khan FU, Khan Z, Rehman Au, Rabbi F, Ahmed N, Fang Y. Perception of surgical teams towards surgical site infections in tertiary care hospital Islamabad, Pakistan. Indian J Surg 2019. 10.1007/s12262-019-01972-7.

2. Anderson DJ, Sexton DJ, Kanafani ZA, Auten G, Kaye KS. Severe surgical site infection in community hospitals: Epidemiology key procedures and the changing prevalence of methicillin-resistant Staphylococcus aureus. Infect Control Hosp Epidemiol 2007; 28(9): 1047-53.

3. Rybak MJ, Lomaestro BM, Rotscahfer JC, Moellering RC, Craig WA, Billeter $M$, et al. Vancomycin therapeutic guidelines: A summary of consensus recommendations from the infectious diseases society of America, the American society of health-system pharmacists, and the society of infectious diseases pharmacists. Clin Infect Dis 2009; 49(3):325-7.

4. Hayat K, Li P, Rosenthal M, Xu S, Chang J, Gillani AH, et al. Perspective of community pharmacists about community-based antimicrobial stewardship programs. A multicenter cross-sectional study from China. Expert Rev Anti Infect Ther 2019; 17(12):1043-1050.

5. Khan Z, Ahmed N, Khan FU, Karataş Y. Utilization pattern of antibiotics and patient care indicators in the teaching hospitals, Islamabad, Pakistan. SN Comprehensive Clinical Medicine 2019; 1:812-6.

Faiz Ullah Khan ${ }^{1,2,3}$, Zakir Khan ${ }^{4}$, Asim-ur-Rehman ${ }^{5}$ and Yu Fang $^{1,2,3}$

${ }^{1}$ Department of Pharmacy Administration and Clinical Pharmacy, School of Pharmacy Xi'an Jiaotong University, Xi'an, China

${ }^{2}$ Center for Drug Safety and Policy Research, Xi'an Jiaotong University, Xi'an, China

${ }^{3}$ The Global Health Institute, Xi'an Jiaotong University, Xi'an, Shaanxi Centre for Health Reform and Development

Research, Xi'an, China

${ }^{4}$ Department of Pharmacology, Institute of Health Sciences, Cukurova University, Adana, Turkey

${ }^{5}$ Department of Pharmacy, Quaid-i-Azam University, Islamabad, Pakistan 
Correspondence to: Prof. Yu Fang, Department of Pharmacy Administration and Clinical Pharmacy, School of pharmacy Xi'an Jiaotong University, Xi'an, China

E-mail: yufang@mail.xjtu.edu.cn
Received: February 14, 2019; Revised: December 12, 2019; Accepted: January 07, 2020

DOI: https://doi.org/10.29271/jcpsp.2020.08.882 